\title{
Resonant-Body Fin-FETs with sub-nW power consumption
}

\author{
Sebastian T. Bartsch, Daniel Grogg, Andrea Lovera, Dimitrios Tsamados, Suat Ayöz, Adrian M. Ionescu \\ Nanoelectronic Devices Laboratory, Ecole Polytechnique Fédéral de Lausanne (EPFL), Lausanne, Switzerland \\ sebastian.bartsch@epfl.ch, adrian.ionescu@epfl.ch, phone: +41 21693 4609/3976
}

\begin{abstract}
This paper presents, for the first time, experimental evidence on resonant-body Fin-FETs (RB-FinFET) with two independent lateral gates, operated from weak to strong inversion, which enables unique trade-off between power consumption and gain. Resonance frequencies from $25 \mathrm{MHz}$ to $80 \mathrm{MHz}$ with quality factors of the order of 3000 and motional resistances of the order of tens of $\mathrm{kOhm}$ are demonstrated with a mixer mode measurement technique, dedicated to ultra-scaled resonators. The power consumption of the active resonators can be reduced in weak inversion of the RB-FinFET well below $1 \mathrm{nW}$, which is a record value compared to any prior active NEM resonator.
\end{abstract}

\section{Introduction}

Mechanical resonators are under intensive study due to their promising applications in ultrasensitive mass sensing [1] and signal generation [2]. Resonators with intrinsic signal amplification mechanisms [3-6] are particularly well-suited to overcome the signal loss associated with nanometer scaling and increasing resonant frequency. Achieving low power consumption and tunable transistor and resonator parameters remain important, depending on its final application, such as interfacing resonators on-chip with advanced CMOS integrated circuitry for wireless communications or integrated sensing applications. In previous active MEM resonators [3-6] the dc power consumption was found to be dominant over the ac one, ranging from $100 \mu \mathrm{W}$ to $10 \mathrm{~mW}$.

\section{Fabrication and Detection Technique}

The laterally vibrating Fin FET resonator investigated in this work is a NEM device laterally actuated by two independent gate electrodes via $120 \mathrm{~nm}$ electrostatic transduction air gaps defined by e-beam lithography on thin film SOI substrate. The buried oxide is used as sacrificial layer to release mechanically movable parts. The detailed fabrication process is reported elsewhere [7]. Figs. 1a-b show SEM images of representative RB-FinFET devices with sub-micron cross sections and a fin height of $200 \mathrm{~nm}$ defined by the silicon film thickness. The length of the clamped vibrating beam is always longer than the transistor channel length. Note that in Fig. 1b the cross-sectional SEM image is taken after focused ion beam (FIB) cut and show the resonator prior to the final poly-Si release. Table 1 reveals the

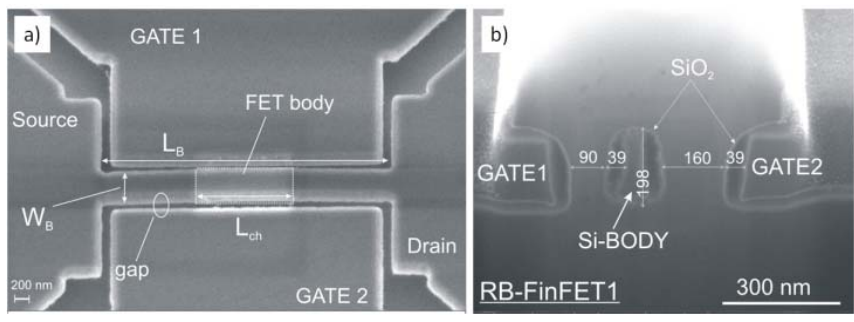

Fig. 1: (a) SEM top-view of a fabricated RB-FinFET with relevant dimensions indicated, (b) cross-sections of RB-FinFET 1. The fin height is given by the silicon film thickness $(\sim 200 \mathrm{~nm})$, the fin width is defined by lithography and thermal oxidation.

\begin{tabular}{|l|l|l|l|}
\hline & RB-FinFET 1 & RB-FinFET 2 & RB-FinFET 3 \\
\hline Beam length $\mathrm{L}_{\mathrm{B}}$ & $3 \mu \mathrm{m}$ & $10 \mu \mathrm{m}$ & $6 \mu \mathrm{m}$ \\
\hline Beam width $\mathrm{W}_{\mathrm{B}}$ & $\mathbf{1 5 0} \mathbf{~ n m}$ & $\mathbf{4 4 0} \mathbf{~ n m}$ & $\mathbf{4 4 0} \mathbf{~ n m}$ \\
\hline FET width $\mathrm{W}_{\mathrm{ch}}$ & $200 \mathrm{~nm}$ & $200 \mathrm{~nm}$ & $200 \mathrm{~nm}$ \\
\hline FET length $\mathrm{L}_{\mathrm{ch}}$ & $1 \mu \mathrm{m}$ & $1 \mu \mathrm{m}$ & $1 \mu \mathrm{m}$ \\
\hline Actuation gap & $90-160 \mathrm{~nm}$ & $120 \mathrm{~nm}$ & $120 \mathrm{~nm}$ \\
\hline Oxide thickness & $40 \mathrm{~nm}$ & $40 \mathrm{~nm}$ & $40 \mathrm{~nm}$ \\
\hline Designed $f_{\text {res }}$ & $170 \mathrm{MHz}$ & $38 \mathrm{MHz}$ & $106 \mathrm{MHz}$ \\
\hline Experim. $f_{\text {res }}$ & $\mathbf{7 9} \mathbf{M H z}$ & $\mathbf{2 5} \mathbf{M H z}$ & $\mathbf{7 0 ~} \mathbf{M H z}$ \\
\hline
\end{tabular}

Table 1: Parameters of designed and fabricated RB-FETs.

dimensions of three devices under test in this work. Fig. 2a shows the schematic set-up for RF characterization. Lock-in amplifier detection used here is applied for the accurate investigation of ultra-scaled RB-FinFET resonators: it enables the measurement of very small output signals at high resonant frequencies, when the transistor is biased from weak to strong inversion (therefore, when the transistor transconductance varies over a few decades).

To detect the motion of the suspended FinFET on resonance, we rely on the nonlinear of the current-gate voltage dependence of the transistor, using it as heterodyne mixer [8]. On the FinFET gate, an RF signal is applied, superimposed on $\mathrm{V}_{\mathrm{G} 2}$. The mixing occurs by injecting a second, high level $\mathrm{RF}$ current (denoted as LO in Fig, 2) at the drain. The nonlinearity in $\mathrm{I}_{\mathrm{D}}\left(\mathrm{V}_{\mathrm{G} 2}\right)$ results in various linear combinations of the original frequencies (and harmonics) of these two inputs. The low-frequency output component at $\omega_{i f}=$ $\left|\omega-\omega_{l o}\right|$ carries then the high-frequency properties of the resonator. A mixer measurement suppresses the parasitic effect of the feed-through capacitance $C_{f}$ (indicated in a small signal equivalent circuit [9] of Fig. 2b), and the phase sensitive signal recovery with lock-in amplifier increases sensitivity. 

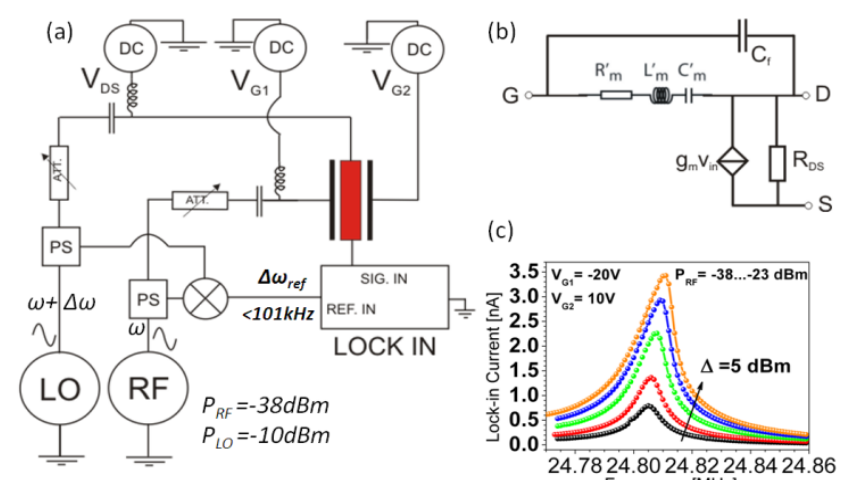

(c)

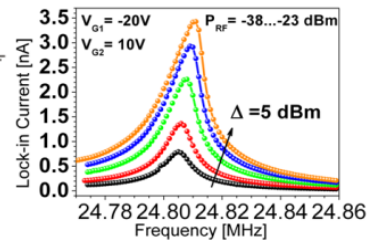

Fig. 2: (a) Two-source mixer set-up with lock-in amplifier in ac-coupled current mode. Typical power levels are indicated, with an if-frequency below $101 \mathrm{kHz}$. (b) Simplified small-signal equivalent circuit of a RB-FET, with the parasitic feed-through capacitance $\mathrm{C}_{\mathrm{f}}$ indicated. (c) With increasing $r f$-power, the resonance is driven into mechanical non-linearity.

Within the small-signal approximation at a given bias point, we can derive the mixing current [10] to follow equation (1):

$\tilde{\imath}_{\text {mix }}\left(\omega_{i f}\right) \sim\left(\tilde{g}_{m} \tilde{v}_{D S}+\frac{d g_{D S}}{d V_{G 2}} \frac{\tilde{C}_{t o t}}{C_{t o t}} \cdot V_{G 2} \tilde{v}_{D S}\right) \cos \left(\omega_{i f} t\right)$

where $\tilde{v}_{D S}$ is the ac signal applied on the drain terminal and $g_{D S}$ the FET output conductance. The current modulation in the transistor results from the field effect with the applied gate RF signal, expressed in the small-signal transconductance $\tilde{g}_{m}$, on one hand (left addend), and from the change of the total capacitance $\tilde{C}_{\text {tot }}$ due to the motion of the beam on the other hand (right addend). The latter is comprised by the air-gap, the oxide and the depletion capacitance.

The ac power levels are set to operate in the mechanically linear region (see Fig. 2c). The electrical characterization is performed in a Süss Microetec prober chamber, under vacuum $\left(\sim 10^{-5} \mathrm{mbar}\right)$ and at room temperature $(300 \mathrm{~K})$.

\section{Experimental Results}

\section{A. Static characteristics:}

Figs. 3-6 shows the transfer $\left(\mathrm{I}_{\mathrm{D}}-\mathrm{V}_{\mathrm{G} 2}\right)$ and output $\left(\mathrm{I}_{\mathrm{D}}-\mathrm{V}_{\mathrm{D}}\right)$ characteristics of double-gate RB-FinFETs. Throughout this work, the gate voltage is applied asymmetrically, i.e. $\mathrm{V}_{\mathrm{G} 1}$ is set constant while $\mathrm{V}_{\mathrm{G} 2}$ is swept over a range of values. The FET is designed to work as an enhancement mode device with a designed threshold voltage $\mathrm{V}_{\text {th }}$ in the order of a few volts. The $\mathrm{V}_{\text {th }}$ shift to lower values is likely caused by charges in/on the gate oxide during processing. The FET-body can be fully depleted by the action of one (RB-FinFET 1) or both lateral gates (RB-FinFET 2 and 3), depending on the width of the fin. Fig. 3 highlights the tuning of the threshold voltage with $\mathrm{V}_{\mathrm{G} 1}$, which is extrapolated at maximum transconductance. It varies linearly (inset, Fig. 3) as expected in a fully depleted case. Fig. 5 and 6 show the $\mathrm{I}_{\mathrm{D}}-\mathrm{V}_{\mathrm{G} 2}$ data of RB-FinFET 1 and 2, respectively. At $\mathrm{V}_{\mathrm{G} 1}=$ $-20 \mathrm{~V}$, the threshold voltage is shifted to positive values. In contrast to reports on accumulation NEM devices [3], enhancement-mode SG-FETs with poor sub-threshold leakage and dielectric transduction resonating transistors [5], we achieve in our RB-FinFETs clear exponential dependence of $I_{d}$ on $V_{g}$ in the weak inversion region and very low $I_{\text {off }}$ $\left(\sim 10^{-13} \mathrm{~A}\right)$, enabling its exploration at resonance. The FET saturates at current levels of several $\mu \mathrm{A}$. The red points along the transfer characteristic indicate the bias conditions at which the mechanical resonance is later observed.

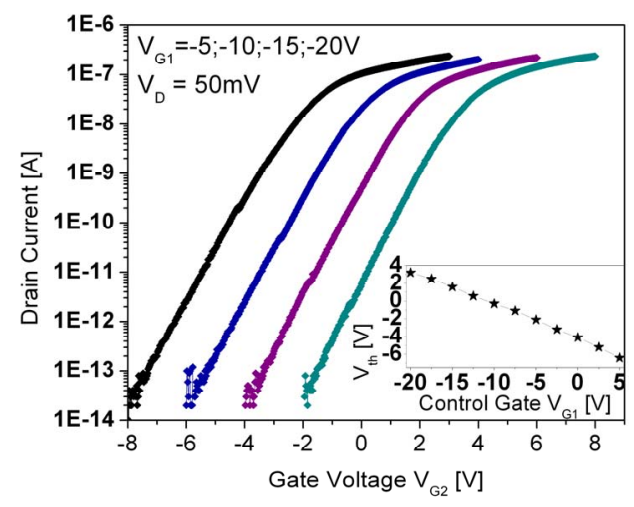

Fig. 3: Experimental $I_{D}-V_{G 2}$ characteristics of RB-FinFET 1. The threshold voltage can be shifted linearly with the voltage $\mathrm{V}_{\mathrm{G} 1}$ (inset).

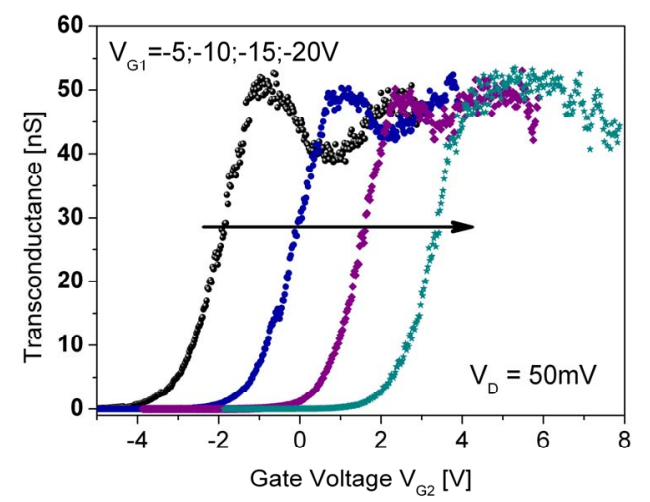

Fig. 4: Transconductance vs. $V_{\mathrm{G} 2}$ of RB-FinFET 1, with independent gate $\mathrm{V}_{\mathrm{G} 1}$ as parameter.

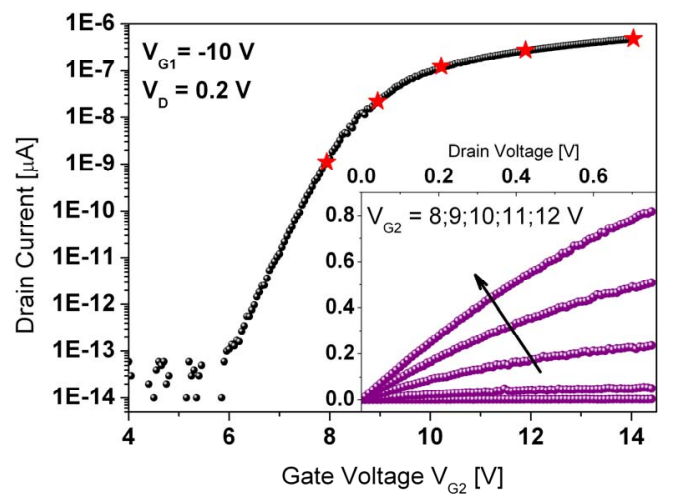

Fig. 5: Experimental $I_{D}-V_{G 2}$ characteristics of RB-FinFET 1 (taken on a different wafer die). The red stars indicate the FET point of operation at which resonance is observed. The inset shows the FET output characteristics $\mathrm{I}_{\mathrm{D}}-\mathrm{V}_{\mathrm{D}}$. 


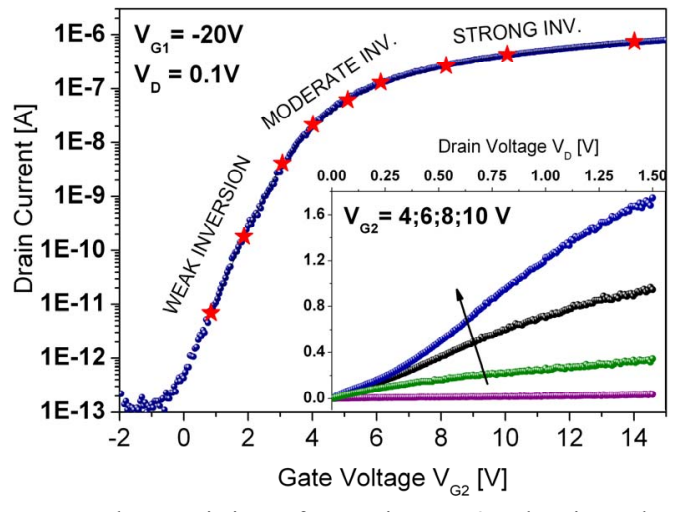

Fig. 6: $I_{D}-V_{G 2}$ characteristics of RB-FinFET 2, showing clear weak, moderate and strong inversion regions and very low $\mathrm{I}_{\text {off. }}$. The $\mathrm{V}_{\text {th }}$ is around 5 $\mathrm{V}$.

\section{B. Resonance in mixer and direct transmission:}

Figs. 7-8 show the transmission response of RB-FinFET 2. The RF-signal is added to $\mathrm{V}_{\mathrm{G} 1}$, and $\mathrm{V}_{\mathrm{G} 2}$ enables the tuning of the FET characteristics. It is emphasized that the dc and RF characteristics performed on the same device under equivalent conditions are very coherent, showing a decrease of the resonance peak when the gate bias moves from strong to weak inversion. We expect the mixing current (highest at resonance) to be proportional to the device transconductance. This can be readily inferred from Fig. 7 ( $\mathrm{g}_{\mathrm{m}}$ data smoothed), which compares the transistor transconductance with the measured peak if-current at each bias point. The inset shows the peak if-current plotted against the transconductance in a $\log -\log$ scale, which varies quasi-linearly with the transconductance, as expected in sub-threshold. The mechanical resonance can now be correlated with a specific bias point of the suspended FinFET. Figs. 7a shows the transmission of RB-FinFET 2 with (loaded) quality factors in the range 2800 to 3000 . The resonant peaks from left to right follow the transfer characteristic of the same transistor. The frequency increases as the electrostatic field decreases (spring softening). Fig. $7 \mathrm{~b}$ demonstrates the resonating FinFET in weak inversion. This corresponds to a static power

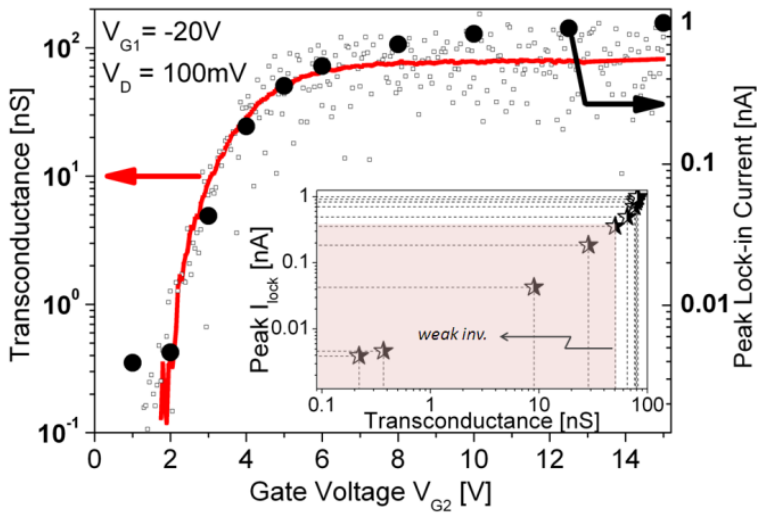

Fig. 7: Transconductance $g_{m}$ and peak lock-in current vs. $V_{G 2}$. The inset shows the correlation of the lock-in peak current and the RB-FinFET transconductance, $g_{\mathrm{m}}$.
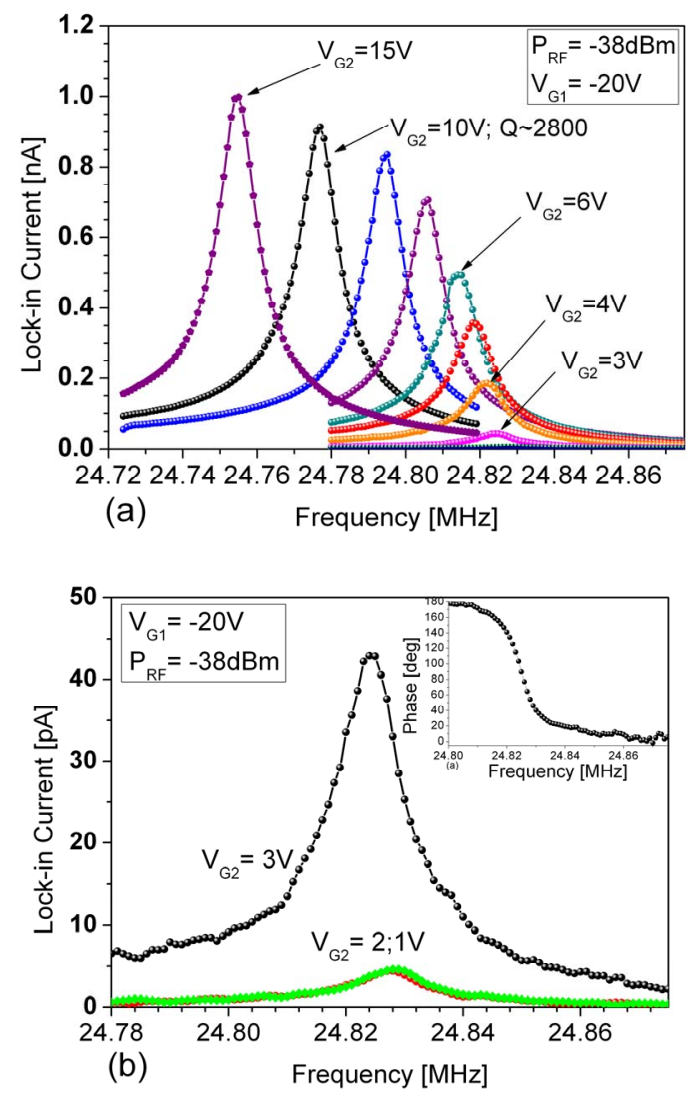

Fig. 8: (a) Transmission characteristic of RB-FinFET 2, with $\mathrm{V}_{\mathrm{G} 2}$ as parameter from strong to weak inversion, according to the red points indicated in Fig.6. (b) Resonance of RB-FinFET 2 in weak inversion. The inset shows the $180^{\circ}$ phase change at resonance (for $\mathrm{V}_{\mathrm{G} 2}=3 \mathrm{~V}$ ).

consumption of $300 \mathrm{pW}$, considering a given $\mathrm{V}_{\mathrm{G} 2}=3 \mathrm{~V}$, $\mathrm{V}_{\mathrm{D}}=100 \mathrm{mV}$ and $\mathrm{g}_{\mathrm{m}}=10 \mathrm{nS}$. We note that the high output impedance associated with the sub-threshold driven FET limits the available signal level due to the mismatch towards the $1 \mathrm{k} \Omega$ input impedance of the lock-in detector.

Fig. 9 shows similar results for the $150 \mathrm{~nm}$ wide FinFET. The discrepancy between designed and measured resonance (compare Table 1) arise from the reduced silicon to silicon oxide ratio for the more scaled mechanical resonator. The lower Young's module of $\mathrm{SiO}_{2}$ results in an overall lower quality factor $(\mathrm{Q}<2000)$. At $80 \mathrm{MHz}$, the resonance of the FinFET in weak inversion can be detected (see Fig. 8 inset). A third RB-FinFET with a resonance frequency of $\sim 70 \mathrm{MHz}$ was measured using direct and mixer mode measurement techniques and comparable results are obtained (Fig. 10a-b) in strong inversion. The sub-threshold resonance characteristics observed with the mixer mode measurement cannot be obtained using the direct measurement technique: any transmitted signals become too small due to the high impedance of the RB-FinFET in weak-inversion when compared to the impedance of the measurement equipment $(50 \Omega)$. However, the comparison of the results allows the empirical calculation of device conversion transconductance 


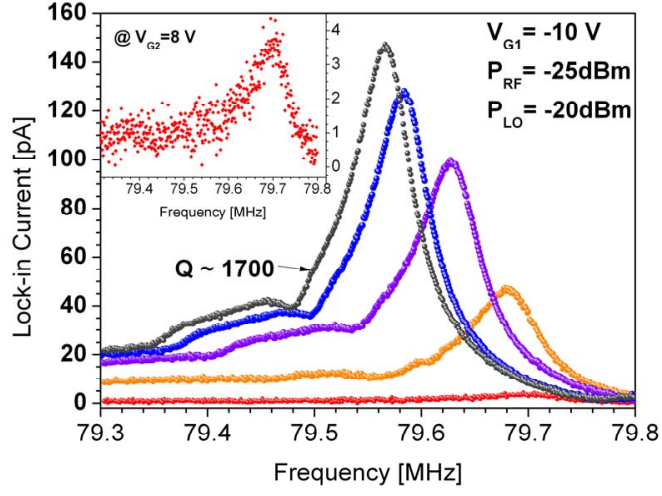

Fig. 10: Transmission characteristic of RB-FinFET 1, with $V_{\mathrm{G} 2}$ as parameter from strong to weak inversion, according to the red points indicated in Fig. 5. The inset shows the frequency response in weak inversion. Albeit close to the noise floor, the peak is still detectable.
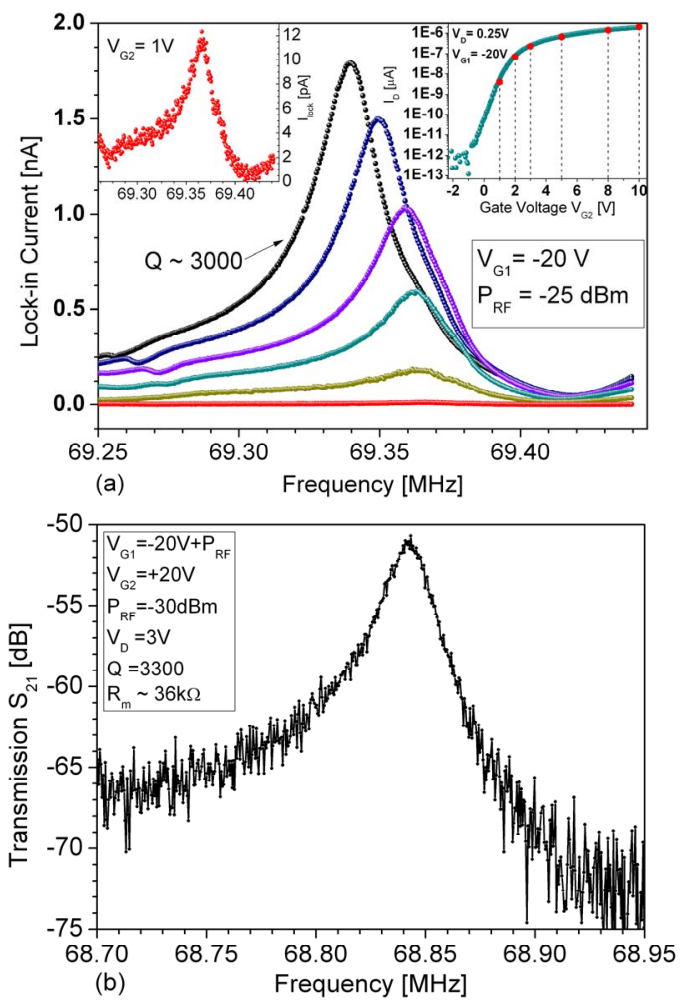

Fig. 11: Transmission response in a mixer (a) and direct (b) measurement of a 70MHz RB-FinFET3. Note that (a) and (b) have same device design, but are measured on a different wafer die.

and hence the equivalent circuit parameters. A motional resistance value of $36 \mathrm{k} \Omega$ is calculated for the $70 \mathrm{MHz}$ resonator $\left(\mathrm{V}_{\mathrm{D}}=3 \mathrm{~V}\right)$.

\section{Power-gain trade-off}

In Fig. 12, the design space for the transconductance and $\mathrm{dc}$ power consumption is depicted for three orders of magnitudes of $g_{m}$ and six orders of magnitude of $P_{d c}$ values, providing a significant selection range of operating points in sub- $\mu \mathrm{W}$ levels for the designers of ultra-low power systems.

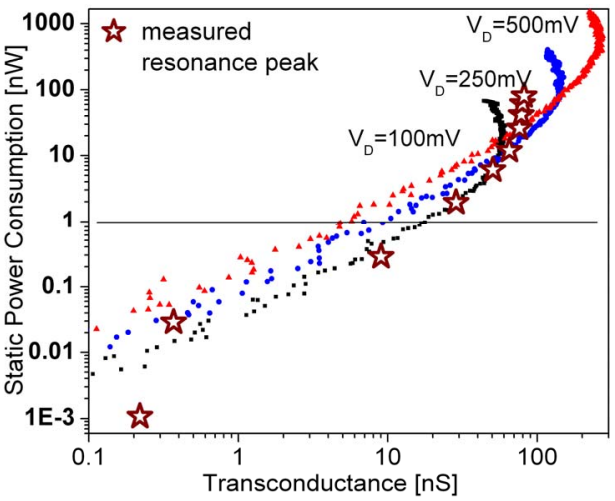

Fig. 12: The power-gain trade-off with $V_{D}$ as parameter. The stars indicate the equivalent points of operation of RB-FinFET 2 at which experimental resonance peaks were observed.

\section{Conclusion}

We have demonstrated the operation of resonant body FinFETs in the sub-threshold region using a mixer technique and the possibility to obtain DC power levels as small as $300 \mathrm{pW}$ or less. This opens new design windows for interfacing ultra-low power devices with on-chip integrated circuitry and is a key step towards large scale NEM device integration.

\section{Acknowledgements: This work was funded by FP7 European project NEMSIC.}

\section{References}

1. Y.T. Yang, et al., Zeptogram-scale nanomechanical mass sensing. Nano Letters, 2006. 6(4): p. 583-586.

2. X.L. Feng, C.J. White, A. Hajimiri, and M.L. Roukes, $A$ self-sustaining ultrahigh-frequency nanoelectromechanical oscillator. Nature Nanotechnology, 2008. 3(6): p. 342-346.

3. D. Grogg, M. Mazza, D. Tsamados, and A.M. Lonescu, Multi-Gate Vibrating-Body Field Effect Transistor (VB-FETS). Ieee International Electron Devices Meeting 2008, Technical Digest, 2008: p. 663-666 949.

4. C. Durand, et al., In-plane silicon-on-nothing nanometer-scale resonant suspended gate MOSFET for In-IC integration perspectives. Ieee Electron Device Letters, 2008. 29(5): p. 494-496.

5. D. Weinstein and S.A. Bhave, The Resonant Body Transistor. Nano Letters, 2010. 10(4): p. 1234-1237.

6. R.R. He, X.L. Feng, M.L. Roukes, and P.D. Yang, Self-transducing silicon nanowire electromechanical systems at room temperature. Nano Letters, 2008. 8(6): p. 1756-1761.

7 A. Lovera, et al., Active NEM Filters for Communications Applications Based on Vibrating Body Transistors, ESSDERC 2010.

8. R. Knobel, C.S. Yung, and A.N. Cleland, Single-electron transistor as a radio-frequency mixer. Applied Physics Letters, 2002. 81(3): p. 532-534.

9 D. Grogg, et al., Small Signal Modeling of Charge and Piezoresistive Modulations in Active MEM Resonators, ESSDERC 2009.

10. S. Rosenblatt, et al., Mixing at $50 \mathrm{GHz}$ using a single-walled carbon nanotube transistor. Applied Physics Letters, 2005. 87(15). 